\title{
Dual-Color Photodetection Based on Speed- Differentiated Photoresponse with High Photogain
}

Qi Yang ${ }^{\dagger, \xi, \perp}$, Jun Shen*, $, \xi, \perp$, Hao Jiang ${ }^{\dagger}$, Chongqian Leng ${ }^{\dagger}$, Zhisheng Zhang ${ }^{\dagger}$,

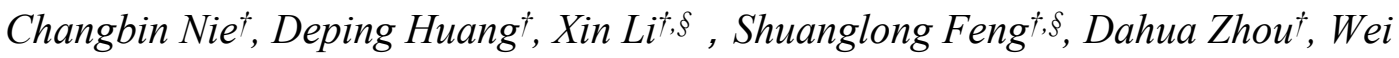
$\mathrm{Luo}^{\dagger}$, Wei Yao ${ }^{\dagger}$, Lu Wang ${ }^{\dagger}$, Ling Sun ${ }^{\dagger}$, and Xingzhan Wei ${ }^{\dagger}, \xi$

\section{Supporting Information}

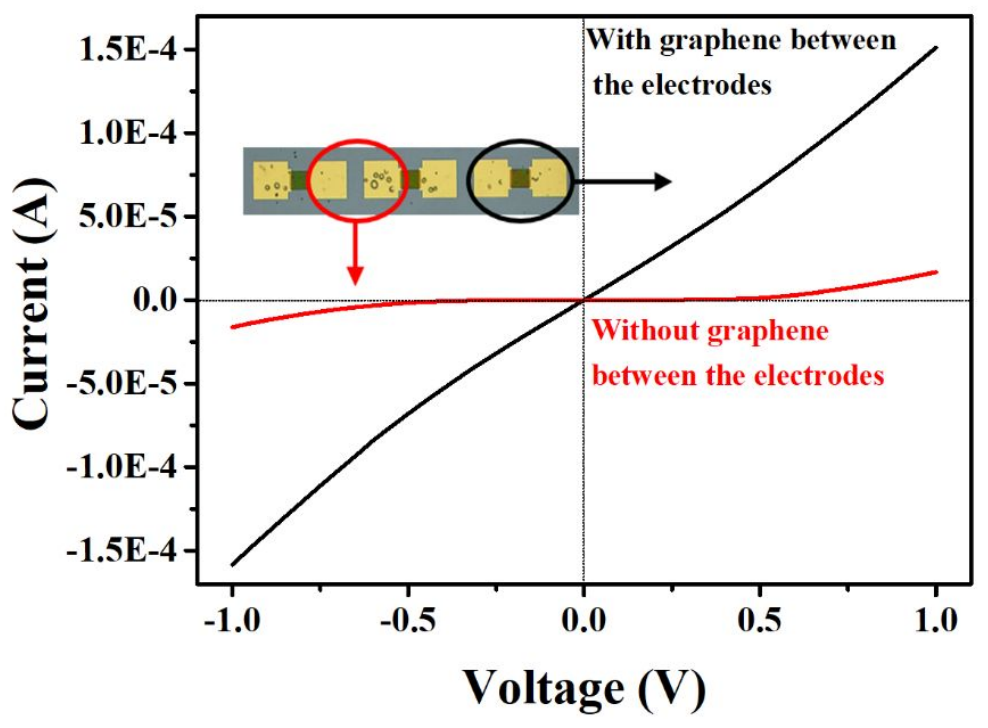

Figure S1. The isolation effect after adding $\mathrm{SiO}_{2}$ between the InGaAs and $\mathrm{Au}$ electrodes. The IV curve test has good conduction between two Au electrodes with graphene, and the IV curve test between two Au electrodes without graphene is basically insulated. 

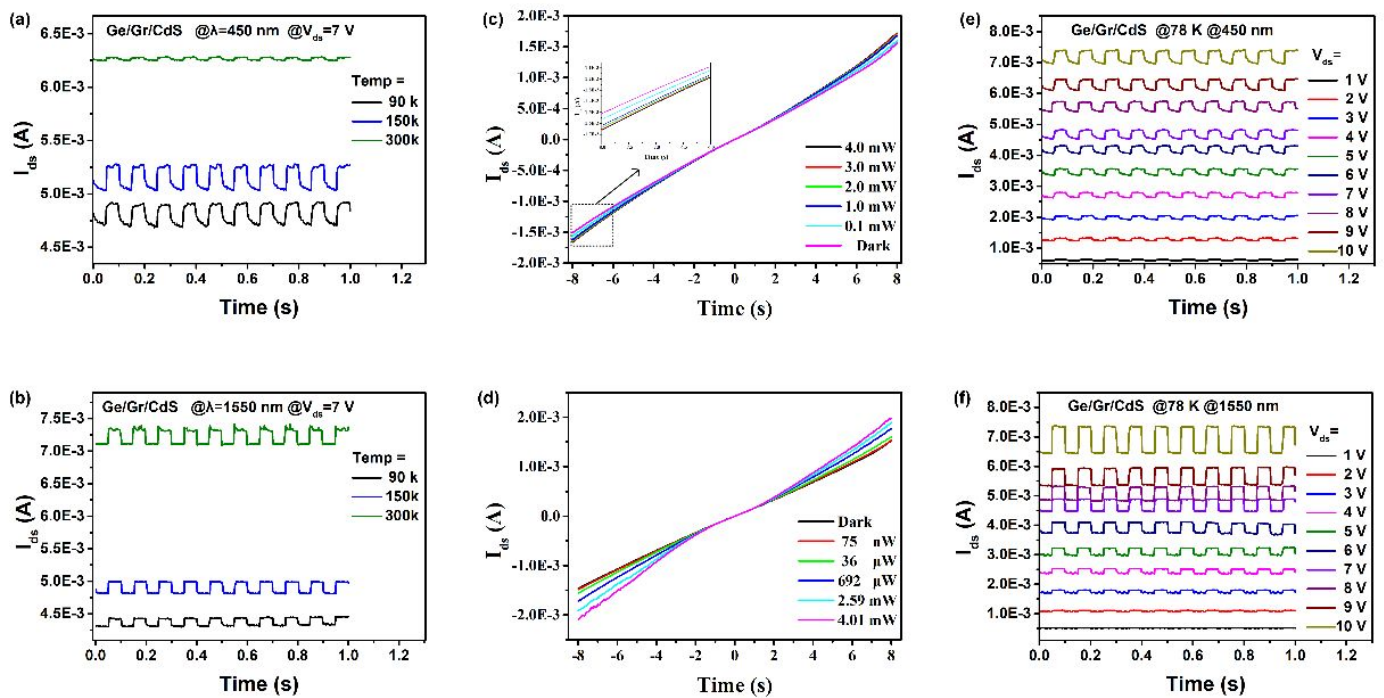

Figure S2. Comparison of photocurrents of Ge / Graphene / CdS detectors under two wavelength light sources (a), (b) at different temperatures (c), (d) at different light illumination powers (e), (f) different source-drain voltages

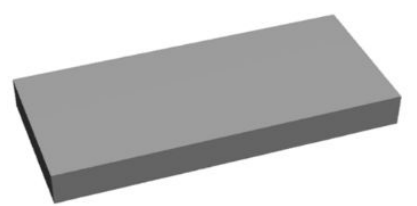

InP Substrate

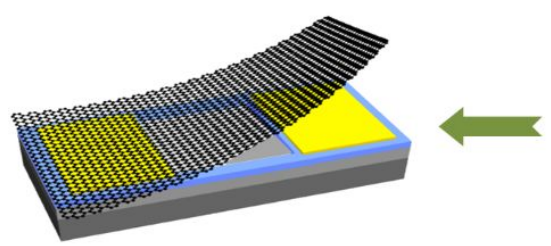

Graphene Transferring

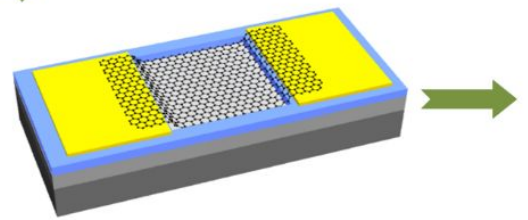

Graphene Patterning

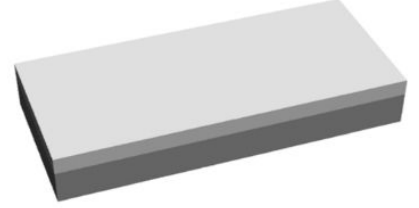

InGaAs Epitaxy

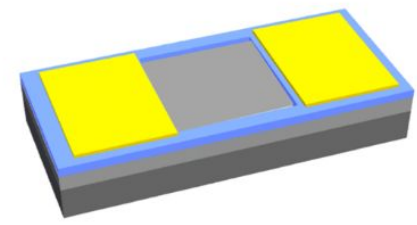

DHF Cleaning

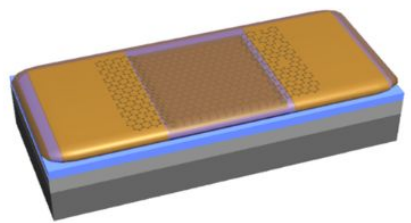

CdS Deposition
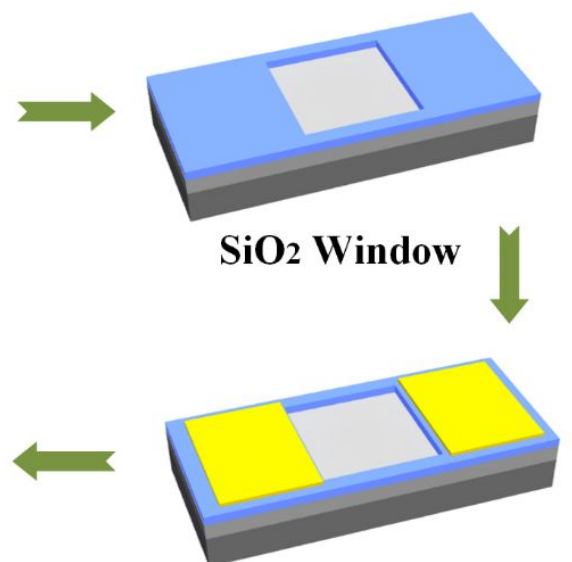

Electrodes Patterning

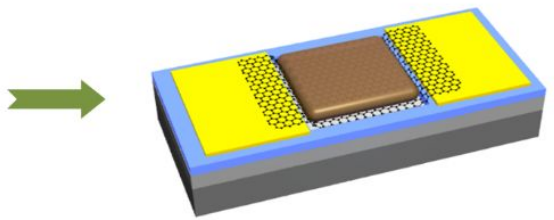

CdS Patterning

Figure S3. Schematic diagram of the device processing flow. 


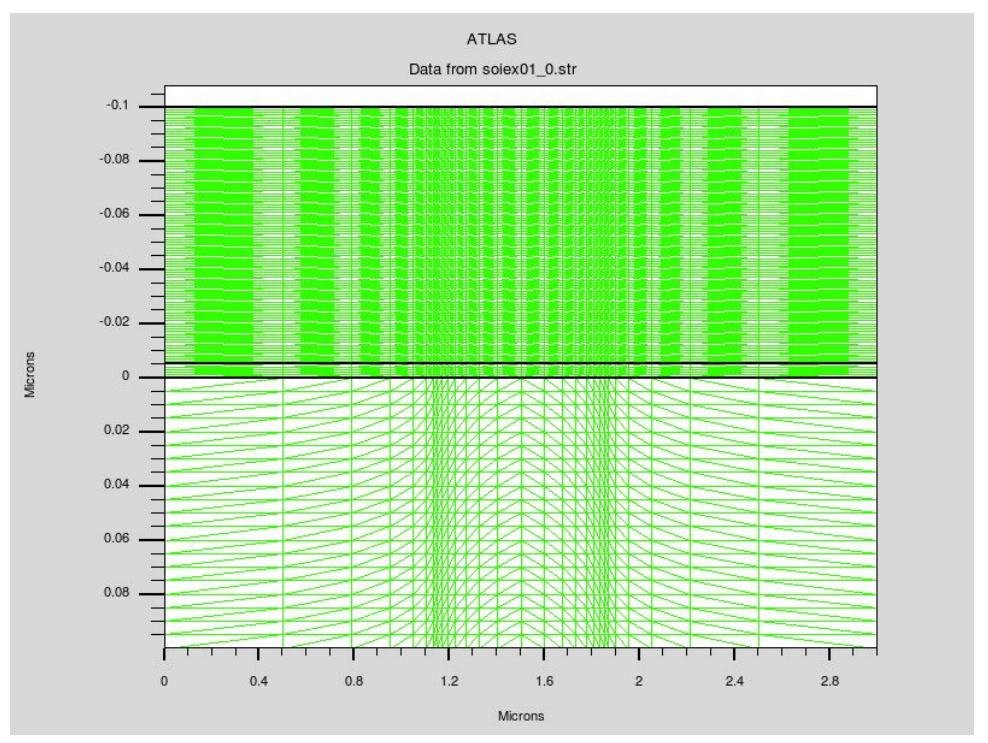

Figure S4. Meshing diagram for device simulation.

Table S1. Basic parameters of the graphene model in Silvaco

\begin{tabular}{|c|c|c|c|c|c|c|}
\hline Parameter & $\begin{array}{c}\mathbf{E}_{\mathbf{g}} \\
(\mathbf{e V})\end{array}$ & $\chi$ & $\begin{array}{c}\boldsymbol{\mu}_{\mathbf{n}} \\
\left(\mathbf{c m}^{2} / \mathbf{V}-\mathbf{s}\right)\end{array}$ & $\begin{array}{c}\boldsymbol{\mu}_{\mathbf{p}} \\
\left(\mathbf{c m}^{\mathbf{2}} / \mathbf{V} \cdot \mathbf{s}\right)\end{array}$ & $\begin{array}{c}\mathbf{X} \\
(\mathbf{K g} / \mathbf{m o l e})\end{array}$ & $\begin{array}{c}\text { Concentration } \\
\left(/ \mathbf{c m}^{2}\right)\end{array}$ \\
\hline description & $\begin{array}{c}\text { Band } \\
\text { gap }\end{array}$ & Permittivity & $\begin{array}{c}\text { Electron } \\
\text { mobility }\end{array}$ & $\begin{array}{c}\text { Hole } \\
\text { mobility }\end{array}$ & Affinity & $\begin{array}{c}\text { Carrier } \\
\text { concentration }\end{array}$ \\
\hline quantity & 0 & 25 & 10000 & 4000 & 4.248 & $1 \mathrm{e} 12$ \\
\hline
\end{tabular}

Table S2. Basic parameters of the CdS model in Silvaco

\begin{tabular}{|c|c|c|c|c|c|c|}
\hline Parameter & $\begin{array}{c}\mathbf{E}_{\mathbf{g}} \\
(\mathbf{e V})\end{array}$ & $\chi$ & $\begin{array}{c}\boldsymbol{\mu}_{\mathbf{n}} \\
\left(\mathbf{c m}^{\mathbf{2}} / \mathbf{V}-\mathbf{s}\right)\end{array}$ & $\begin{array}{c}\boldsymbol{\mu}_{\mathbf{p}} \\
\left(\mathbf{c m}^{2} / \mathbf{V} \cdot \mathbf{s}\right)\end{array}$ & $\begin{array}{c}\mathbf{X} \\
(\mathbf{K g} / \mathbf{m o l e})\end{array}$ & $\begin{array}{c}\text { Concentration } \\
\left(/ \mathbf{c m}^{2}\right)\end{array}$ \\
\hline Description & $\begin{array}{c}\text { Band } \\
\text { gap }\end{array}$ & Permittivity & $\begin{array}{c}\text { Electron } \\
\text { mobility }\end{array}$ & $\begin{array}{c}\text { Hole } \\
\text { mobility }\end{array}$ & Affinity & $\begin{array}{c}\text { Carrier } \\
\text { concentration }\end{array}$ \\
\hline Quantity & 2.4 & 10 & 100 & 25 & 4.5 & $2.2 \mathrm{e} 18$ \\
\hline
\end{tabular}

